\title{
TIPIFICAÇÃO GENÉTICA, ATRAVÉS DA TÉCNICA DE SPOLIGOTYPING, DE ISOLADOS DE MYCOBACTERIUM BOVIS EM ANIMAIS ABATIDOS NA REGIÃO METROPOLITANA DE SALVADOR, BAHIA, BRASIL
}

\section{A.C.F. Costa ${ }^{1}$, N.S. Silva ${ }^{1}$, V.C.M. Rocha ${ }^{2}$, C.A. Rosales Rodriguez ${ }^{2}$, A. Estrela-Lima ${ }^{3}$, E.L.T. Moreira ${ }^{3}$, C. Madruga ${ }^{3}$, S.M. Arruda ${ }^{4}$; J.S. Ferreira Neto ${ }^{2}$, M.C.A. Silva ${ }^{1}$, E.M. de D. Oliveira ${ }^{1}$}

${ }^{1}$ Universidade Federal da Bahia, Escola de Medicina Veterinária, Departamento de Medicina Preventiva, Av. Adhemar de Barros, 500, CEP 40110-1170, Salvador, BA, Brasil. E-mail:mcasilva@ufba.br

\section{RESUMO}

\begin{abstract}
A tuberculose é uma enfermidade infecciosa crônica, que afeta mamíferos e aves e constitui um sério problema de saúde pública e animal. Objetivando realizar um levantamento molecular da enfermidade em bovinos abatidos em matadouros frigoríficos no Estado da Bahia, Brasil, foram analisadas as lesões pulmonares e de linfonodos mediastínicos de 43 carcaças de animais abatidos em três matadouros-frigoríficos localizados na Região Metropolitana de Salvador, Bahia. Sete isolados de Mycobacterium bovis foram identificados, através da técnica do spolygotyping, e discriminados em três diferentes espoligotipos (SB1055, SB0120 e SB0268) descritos no Brasil e em diversas áreas do mundo. Os resultados indicam que o método de diagnóstico utilizado pode contribuir para a criação de uma base de dados para o estudo epidemiológico da tuberculose bovina no Estado da Bahia.
\end{abstract}

PALAVRAS-CHAVE: Bovino, Mycobacterium bovis, spoligotyping, Bahia.

\section{ABSTRACT}

USE OF SPOLIGOTYPING FOR GENETIC TYPING OF MYCOBACTERIUM BOVIS ISOLATES FROM SLAUGHTERED ANIMALS IN THE METROPOLITAN AREA OF SALVADOR, BAHIA, BRAZIL. Tuberculosis is an infectious chronic disease that affects mammals and birds and constitutes a serious problem for public and animal health. Pulmonary and mediastinic lymph node lesions of 43 animals slaughtered in 3 slaughterhouses in the metropolitan region of the city of Salvador, Bahia, Brazil, were analyzed with the objective of obtaining a molecular survey of the disease in bovines slaughtered in slaughterhouses in the state. Seven isolates of Mycobacterium bovis were identified through the spoligotyping technique and classified into 3 different spoligotypes (SB1055, SB0120, SB0268), described in Brazil and in many areas worldwide. The results indicate that the diagnostic method utilized may contribute to the creation of a database for the epidemiologic study of bovine tuberculosis in the state of Bahia.

KEY WORDS: Bovine, Mycobacterium bovis, spoligotyping, Bahia.

\section{INTRODUÇÃO}

A tuberculose causada pelo Mycobacterium bovisé uma doença infecto-contagiosa de caráter zoonótico e evolução crônica que acomete principalmente bovinos e bubalinos. Caracteriza-se pelo desenvolvimento progressivo de lesões nodulares denominadas tu- bérculos, que podem se localizar em qualquer órgão ou tecido (Brolio, Lima Filho, 1976).

As graves consequências desta zoonose não residem exclusivamente nos significativos coeficientes de morbidade e mortalidade para o homem, mas também nos prejuízos econômicos, decorrentes de sua disseminação nas populações animais, as quais,

\footnotetext{
${ }^{2}$ Universidade de São Paulo, Faculdade de Medicina Veterinária e Zootecnia, Departamento de Medicina Veterinária Preventiva e Saúde Animal, São Paulo, SP, Brasil.

${ }^{3}$ Universidade Federal da Bahia, Escola de Medicina Veterinária, Departamento de Patologia e Clínicas, Salvador, BA, Brasil.

${ }^{4}$ Fundação Osvaldo Cruz, Centro de Pesquisas Gonçalo Muniz, Salvador, BA, Brasil.
} 
por seu turno, assumem importante papel como fontes de infecção para a população humana (AcHA; SZYFRES, 1977).

A importância econômica atribuída à doença bovina está baseada nas perdas diretas resultantes da morte de animais, da queda no ganho de peso e diminuição da produção de leite, do descarte precoce e eliminação de animais de alto valor zootécnico e condenação de carcaças ao abate. Estima-se que os animais infectados percam de 10 a $25 \%$ de sua eficiência produtiva. Existe ainda a perda de prestígio e credibilidade da unidade de criação onde a doença é constatada (BRASIL, 2006).

$\mathrm{O}$ agente etiológico da tuberculose pertence ao gênero Mycobacterium que contem mais de 50 espécies. OM. bovis está agrupado no "ComplexoMycobacterium tuberculosis", representado pelas principais micobactérias com importância epidemiológica: $M$. tuberculosis, M. bovis, M. microti eMycobacterium canetti, M. africanum, este último ainda não isolado no Brasil (BIER, 1978; CORNER, 1994; BROSCH et al., 2002; ZINK; Nerlich, 2004). Novas espécies, a exemplo da $M$. caprae e M. pinnipedii, têm sido propostas para inclusão neste complexo (Cousins et al., 2003).

No Brasil, a situação da tuberculose bovina não se encontra bem delineada, pois os estudos disponíveis são escassos e não sistematizados. Dados epidemiológicos oficiais fornecem índices de prevalência da doença de 3,3\% e 1,2\% para os períodos de 1967 a 1976 e de 1988 a 1992, respectivamente, e de $1,3 \%$ de animais infectados no período de 1989 a 1998 (BRASIL, 2008). Os dados sobre incidência da tuberculose em bovinos também são pouco conhecidos, existe uma estimativa de ocorrência em torno de $5,7 \%$ do rebanho nacional. Destes, $0,14 \%$ corresponde ao achado de lesões em matadouros (Pustiglione Neto, 1997; Roxo, 2000).

Na Bahia, estudos realizados no Município de Ilhéus-Bahia, avaliaram, por meio do teste intradérmico cervical comparado, a prevalência da tuberculose na região, constatando a presença de animais positivos em 10,6\%, das 85 propriedades estudadas, e uma prevalência de 2,8\%, dos 916 animais testados (RIBEIRO et al., 2003). Na bacia leiteira de Alagoinhas-Bahia foi observada uma taxa de $5,5 \%$ de animais positivos ao teste cervical comparado, econdições favoráveis para disseminação da tuberculose na população humana pelo hábito do consumo de leite "in natura" (Costa et al., 1995/1996).

O principal método de diagnóstico da tuberculose em bovinos, in vivo, preconizado pelo Programa Nacional de Controle e Erradicação da Brucelose e Tuberculose (PNCEBT), é o teste imunoalérgico que se baseia na reação de hipersensibilidade tardia mediada por linfócitos sensibilizados, deflagrada em indivíduos previamente expostos às tubérculo-proteínas específicas (Roxo, 1996). Já o diagnósticopost-mortem, é realizado pelo exame dos órgãos na necropsia ou achados de abate, as lesões suspeitas são encaminhadas para o exame histopatológico para verificação da presença de granuloma (KANTOR, 1979).

O diagnóstico definitivo é realizado pelo isolamento do agente micobacteriano de lesões ou secreções de animais suspeitos. A identificação do agente é feita por meio de provas bioquímicas, técnicas de biologia molecular, como a reação de polimerase em cadeia (PCR), ou análise química de diferentes grupos funcionais de ácidos micólicos, presentes na parede das micobactérias (Almeida et al., 2004).

Uma combinação que tem se mostrado eficiente para o diagnóstico direto da tuberculose bovina é o isolamento através da bacteriologia clássica, seguido da identificação por métodos moleculares: PCR-PRA (Telenti et al., 1993); Spoligotyping (KAMERBeEK et al., 1997; v ANSOOLINGENET etal.,1995);MIRU-VNTR(SUPPLY et al., 2000). Além de identificar a espécie, as técnicas de Spoligotyping e MIRU-VNTR permitem discriminar isolados de $M$. bovis.

Ospoligotyping possibilita a detecção e tipificação das micobactérias do Complexo M. tuberculosis, sendo indicado como técnica de eleição para comparação de estirpes com poucas cópias de IS6110, além de permitir diferenciação de estirpes de M. bovis eM. tuberculosis (SАКАмото, 2001). Esta técnica baseia-se na amplificação enzimática dolocus DR (DirectRepeat) do Complexo $M$. tuberculosis, ou seja, este método detecta a presença ou ausência de espaçadores no locus DR, diferenciando as amostras isoladas, podendo ser empregado na investigação epidemiológica, permitindo a identificação do curso da infecção e das rotas de transmissão da doença, ou mesmo da compreensão epidemiológica da infecção, que são fundamentais para um melhor controle e erradicação da doença (ROMANO et al., 1996; LiÉBANA et al., 1997; ZANINI et al., 2001; RodRIGUEZ, 2005).

O diagnóstico bacteriológico, mediante isolamento e tipificação da bactéria, é necessário para a vigilância epidemiológica da enfermidade. Nas campanhas de controle ou erradicação, à medida que diminui a prevalência da doença, é mais importante tipificar as bactérias isoladas de lesões de tuberculose, com o objetivo de identificar aquelas que induzem reação cruzada com a tuberculina bovina (RIET-CORREA; GARCIA,2001).

O objetivo do presente trabalho foi utilizar as linhas de inspeção de matadouros-frigoríficos localizados na região metropolitana de Salvador, para diagnosticar a tuberculose bovina e identificar através da técnica de spoligotyping os isolados de Mycobacterium. Este trabalho consiste no primeiro levantamento molecular de tuberculose em bovinos no Estado da Bahia, Brasil. 


\section{MATERIAL E MÉTODOS}

Amostras de pulmão e linfonodos mediastínicos de 43 carcaças bovinas condenadas por tuberculose ou alterações pulmonares de diagnósticos macroscópicos diversos foram coletadas durante a rotina de inspeção em três abatedouros bovinos localizados na Região Metropolitana de Salvador, no período de maio de 2006 a fevereiro de 2007. Dentre os estabelecimentos, um pertencia a rede SIF (Serviço de Inspeção Federal), outro SIE (Serviço de Inspeção Estadual) e outro SIM (Serviço de Inspeção Municipal). As amostras, coletadas em duplicata, foram congeladas e enviadas ao Laboratório de Bacterioses/ EMEV/UFBA, submetidas ao processamento imediato e realização de cultivo.

Cada amostra foi macerada em stomacher, descontaminada pelo método de HPC - Cloreto de1Hexadecilpiridínio (Corner, Trajstaman, 1988; Ambrósio, 2005) e semeadas em meios de LowensteinJensen eStonebrink Leslie (CEntro Panamericanode Zoonosis, 1973) à $37^{\circ} \mathrm{C}$ por até 90 dias. Os cultivos foram observados semanalmente para verificação de crescimento de colônias sugestivas de micobactéria. As colônias isoladas foram submetidas à coloração de Ziehl-Neelsen (CEntro PANAMERICANO DE ZoONOSIS, 1988) e, uma vez confirmada a característica de BAAR (Bacilo álcool-ácido resistente), foi extraído o DNA e enviadas ao Laboratório de Zoonoses Bacterianas/FMVZ/USP para caracterização molecular, por meio da análise de polimorfismo pelo método de spoligotyping executado de acordo com a descrição de KAMERBEEK et al. (1997). Os padrões obtidos na hibridização no spoligotyping foram analisados com aqueles existentes no banco de dados de M. bovis (www.mbovis.org) e publicações (RodriguEz et al., 2004).

\section{RESULTADOS E DISCUSSÃO}

No cultivo, foram isolados BAAR de amostras de dez carcaças $(23 \%)$. Onze (26\%) não apresentaram crescimento durante os 90 dias de cultivo e 22 (51\%) apresentaram contaminação. Das dezamostras BAAR positivas oito cresceram apenas no meio deStonebrink-
Leslie, enquanto que as outras duas cresceram tanto no Stonebrink-Leslie como em meio LowensteinJensen. Dentre as amostras BAAR positivas, seis foram coletas no matadouro municipal e quatro no matadouro estadual.

Os resultados obtidos estãosumariados na Tabela1.

Utilizando a técnica de spoligotyping foi possível identificar polimorfismo entre isolados de $M$. bovis, fator importante a ser considerado na epidemiologia da tuberculose bovina no Estado da Bahia. O BARR SB1055, isolado de maior frequência no estudo, tem ampla distribuição na América Latina, com registros no Brasil, Argentina, Paraguai, Uruguai, México e Costa Rica (www.mbovis.org).

Os espoligotipos SB0120 e SB0268, também identificados no Estado de São Paulo (RodriguEZ, 2005), têm importância em outras regiões do mundo. OSB0268foi encontradoembovinosnaInglaterra (www.mbovis.org) eoSB0120, também denominado deBCG-like(HADDAD etal.,2001), possui grandediversidade dehospedeiros, pois, além de bovinos, já foi isolado de cabras, ovelhas, suínos, animais silvestres e infectando humanos. Este espoligotipo está disseminado em diversos países da Europa, sendo o mais frequente na França (26\%), mas também já foi encontrado nos continentes africano e asiático (www.mbovis.org).

No Reino Unido, Gibson et al., (2004) relataram 50 casos de tuberculose humana por $M$. bovis, surgidos de 1997 a 2000, sendo dois deles provocados pelo espoligotipo SB0120.

Embora a amostragem não tenha seguido uma metodologia apropriada para a análise estatística de casos de tuberculose, com base em categorias de animais, nos frigoríficos de inspeção federal, estadual e municipal, o maior número de micro-organismos BAAR isolados, no frigorífico de inspeção municipal, possivelmente está relacionado com o abate de animais de descartes das pequenas propriedades leiteiras ou mistas, onde a probabilidade de tuberculose aumenta. Além disso, o menor número de animais no abate e a presença do médico veterinário in locu permitem maior rigor e minuciosidade do diagnóstico macroscópico. Valea pena ressaltar que nãoérealizado nenhum exame confirmatório dos animais condenados por tuberculose, nos estabelecimentos supracitados.

Tabela 1- Padrão da espoligotipagem de Mycobacterium bovis isolados de pulmões e linfonodos de bovinos abatidos na região metropolitana de Salvador, Bahia.

\begin{tabular}{llll}
\hline $\begin{array}{l}\text { Espoligotipo segundo } \\
\text { www.mbovis.org }\end{array}$ & Espécie & Perfil & NoAmostras $^{\circ}$ \\
\hline SB1055 & M. bovis & 1100011101111110111111111111111111110100000 & 5 \\
SB0268 & M. bovis & 1101011101001110111110111111111111111100000 & 1 \\
SB0120 & M. bovis & 1101111101111110111111111111111111111100000 & 1 \\
\hline
\end{tabular}




\section{CONCLUSÃO}

A junção das técnicas de diagnósticopost-mortem da tuberculose, preconizadas pelo PNCEBT, com técnicas de genotipagem molecular foi a base do primeiro estudo de identificação das micobactérias encontradas na Região Metropolitana de Salvador, BA, sendo o ponto de partida para a criação de uma base de dados com grande importância para o estudo epidemiológico desta doença no Estado da Bahia.

No presente estudo, pode-se identificar polimorfismo entre os isolados de M. bovis, com base na técnica de spoligotyping, identificando nas amostras pesquisadas qual o espoligotipo de maior frequência, SB1055, corroborando com a hipótese de disseminação desta cepa na América Latina.

\section{REFERÊNCIAS}

ACHA, P.N.; ZYFRES, B. Zoonosis y enfermedades transmisibles comunes al hombre y a los animales. Washington: Organización Panamericana de la Salud, 1977. p.98-111. (Publicación científica, 354).

AMBRÓSIO, S.R.; OLIVEIRA, E.M.D.; ROSALES, C.A.R.; FERREIRA NETO; J.S.N; AMAKU, M. Métodos bacteriológicos aplicados à tuberculose bovina: comparação de três métodos de descontaminação. Arquivos do Instituto Biológico, São Paulo, v.71, p.68, 2004. Suplemento. Trabalho apresentado na REUNIÃO ANUAL DO INSTITUTO BIOLÓGICO, 17.; CONGRESSO DE INICIAÇÃO CIENTÍFICA EM CIÊNCIAS AGRÁRIAS, BIOLÓGICAS E AMBIENTAIS, 2., 2004, São Paulo. Resumo 067. 1CD-ROM.

ALMEIDA, R.F.C.A.; SOARES, C.O.; ARAÚJO, F.R. Tuberculose Bovina: Diagnóstico. In: (Ed.). Brucelose e Tuberculose Bovina: epidemiologia, controle e diagnóstico. Brasília: Empraba Informação Tecnológica, 2004. p.61-80.

BIER, O. Micobactérias. In: (Ed.). Bacteriologia e imunologia. 19.ed. São Paulo: Melhoramentos, 1978. p.585-610.

BRASIL. Ministério da Agricultura, Pecuária e Abastecimento (MAPA). Secretaria de Defesa Agropecuária. Departamento de Defesa Animal. Manual Técnico do Programa Nacional de Controle e Erradicação da Brucelose e Tuberculose (PNCEBT). Brasília, 2006.

BRASIL. Ministério da Agricultura, Pecuária e Abastecimento (MAPA). Instrução Normativa SDA 53 de 12 de julho de 2004. Regulamento técnico do Programa Nacional de Sanidade dos caprinos e Ovinos - PNSCO. Disponível em: <http/www.agricultura.gov.br>. Acesso em: 5 jan. 2008.
BROLIO R., LIMA FILHO M.T. Tuberculose pulmonar. In: VERONESI, R. (Ed.). Doenças infecciosas e parasitárias. Rio de Janeiro: Guanabara Koogan, 317-361, 1976.

BROSCH, R.; GORDON, S.; MARMIESSE, M.; BRODIN, P.; BUCHRIESER, C.; EIGLMEIER, K.; GARNIER, T.; GUTIERREZ, C.; HEWINSON, G.; KREMER, K.; PARSONS, L.M.; PYM, A.S.; SAMPER, S.; VAN SOLLIGEN, D.; COLE, A. A new evolutionary scenario for the Mycobacterium tuberculosis complex. Proccedings of National Academy of sciences of the United States of America, v.99, n.6, p.3684-3689, 2002.

CENTRO PANAMERICANO DE ZOONOSIS. Métodos de laboratório de microbiologia veterinária para el aislamiento e identificacion de micobactérias. Buenos Aires: CPZ, 1973. 48p. (Série monografias científcas y técnicas, 6).

CENTRO PANAMERICANO DE ZOONOSIS. Situación de la tuberculosis bovina em América Latina y el Caribe. Buenos Aires: CPZ, 1988. 23p. (Publicación Especial n.8).

CORNER, L.A. Post mortem diagnosis of mycobacterium bovis infection in cattle. Veterinary Microbiology, v.40, p.53-63, 1994.

CORNER, L.A.; TRAJSTAMAN, A.C. An evaluation of 1- Hexadecylpyridinium Chloride as a decontaminant in the primary isolation of Mycobacterium bovis from bovine lesions. Veterinary Microbiology, v.18, n.2, p.127$143,1988$.

COSTA, J.N.; FREREIRA, M.M.; TEIXEIRA, L. L.; MENEZES, R.V.; RAMALHO, E.J. Tuberculose bovina: estudo preliminar da bacia leiteira de Alagoinhas-Ba. Arquivo da Escola de Medicina Veterinária da Universidade Federal da Bahia, v.18, p.141-148, 1995/1996.

COUSINS, D.V.; BATISTA R, CATALDI A, QUSE V, DOWN S, DUIGNAN P, MURRAY A, DUPONT C, AHMED N, COLLINS DM, BUTLER WR, DAWSON D, RODRIGUEZ D, LOUREIRO J, ROMANO MI, ALITO A, ZUMARRAGA M, BERANRDELLI A. Tuberculosis in seals caused by a novel member of the Mycobacterium tuberculosis complex: Mycobacterium pinnipedii sp. Nov. International Journal of Systematic and Evolutionary Microbiology, v.53, p.1305-1314, 2003.

GIBSON, A.L.; HEWINSON,G.; GOODCHILD, T.; WATT, B.; STORY, A.; INWALD, J.; DROBNIEWKI, F.A. Molecular epidemiology of disease due to Mycobacterium bovis in humans in the United Kingdom. Journal of Clinical Microbiology, v.42, p.431-434, 2004.

HADDAD, N.; OSTYN, A.; KAROUI, C.; MASSELOT, M.; THOREL, M.F.; HUGHES, S.L.; INWALD, J.; HEWINSON, R.G.; DURAND, B. Spoligotype diversity of Mycobacterium bovis strains isolated in France from 1979 to 2000. Journal of Clinical Microbiology, v.39, p.36233632, 2001. 
KAMERBEEK, J.; SCHOULS, L.; KOLK, A.; van AGTERVELD, M.; van SOOLINGEN, D.; KUIJPER, S.; BUNSCHOTEN, A.; MOLHUIZEN, H.; SHAW, R.; GOYAL M.; van EMBDEN J.D. Simultaneous detection and strains differentiation of Mycobacterium tuberculosis for diagnosis and epidemiology. Journal of Clinical Microbiology, v.35, p.907-914, 1997.

KAMERBEEK, J.; SCHOULS, L.; KOLK, A.; VAN AGTERVELD, M.; VAN SOOLINGEN, D.; KUIJPER, S.; BUNSCHOTEN, A.; MOLHUIZEN, H.; SHAW, R.; GOYAL, M.; VAN EMBDEN, J. Simultaneous detection and strain differentiation of Mycobacterium tuberculosis for diagnosis and epidemiology. Journal of Clinical Microbiology, v.35, p.907-914, 1997.

KANTOR, I.N. Bacteriológia de la tuberculosis humana y animal. Martinez: OPAS/OMS. 1979.63p. (Nota Técnica n. 8).

LIÉBANA, E.; ARANAZ, A.; DOMINGUEZ, L.; MATEOS, A.; GONZÁLES- LLAMAZARES, O.; RODRIGUEZ-FERRI, E.F.; DOMINGO,M.; VIDAL, D.; COUSINS, D. The insertion element IS 6110 is a useful tool for DNA fingerprinting of Mycobacterium bovis isolates from cattle and goats in Spain. Veterinary Microbiology, v.54, p.223-233, 1997.

PUSTIGLIONE NETO, L. Tuberculose dos bovinos. Pecuária de corte, São Paulo, v.6, n.66, p.52-53, 1997.

RIBEIRO, A.R.P; LOBATO, F.C.F.; ABREU, V.L.V.; FARIA, E.S.; SILVA, J.A. Prevalência de tuberculose e brucelose bovina no município de Ilhéus. Arquivo Brasileiro de Medicina Veterinária e Zootecnia, v.55, n.1, p.120-122, 2003. (Comunicação).

RIET-CORREA, F.; GARCIA, M. Tuberculose. In: RIET CORREA, F.; SCHILD, A.L.; MENDÉZ, M.C.; LEMOS, R.A.A. (Org). Doenças dos ruminantes e eqüinos. 2. ed. São Paulo, 2001. v.1, p.351-362.

RODRIGUEZ, C.A.R.; ZUMÁRRAGA, M.J.; OLIVEIRA, E.M.; CATALDI, A.A.; ROMANO, M.I.; OTTO, H.H.; BONAFÉ, V.L.; FERREIRA NETO, J.S. Caracterização molecular de isolados de Mycobacterium bovis do Estado de São Paulo Brasil, utilizando a técnica de spoligotyping. Arquivo do Instituto Biológico, São Paulo, v.71, n.1, p.277-282, 2004.

RODRIGUEZ, C.A.R. Sistema de Detecção de focos de tuberculose bovina no Estado de São Paulo utilizando métodos moleculares e epidemiológicos. 2005. 86 f. Tese (Doutorado) Faculdade de Medicina Veterinária e Zootecnia da Universidade de São Paulo, São Paulo, 2005.
ROMANO, M. I.; ALITO, A.; FISANOTI, J. C.; BIGI, F.; KANTOR, I.; CICUTA, M. E.; CATALDI, A.

Comparison of different genetic markers for molecular epidemiology of bovine tuberculosis. Veterinary Microbiology, v.50, p.59-71, 1996.

ROXO, E. Tuberculose bovina: revisão. Arquivos do Instituto Biológico, São Paulo, v.63, n.2, p.91-97, 1996.

ROXO, E. Tuberculose bovina: revisão. Pecuária de corte, v.11, n.101, p.45-50, 2000.

SAKAMOTO, S.M. Tipificação de Mycobacterium bovis por Spoligotyping e por análise de número variável de repetições seriadas (VNTR). 2001. 38f. Tese (Doutorado) - Faculdade de Medicina Veterinária e Zootecnia da Universidade de São Paulo, São Paulo, 2001.

SUPPLY, P.; MAZARS, E.; LESJEAN, S.; VICENT, V.; GICQUEL, B.; LOCHT, C. Variable human minisatellite-likeregions in the Mycobacterium tuberculosis genome. Molecular Microbiology, v.36, n.3, p.762-771, 2000.

TELENTI, A.; MARCHESI, F.; BALZ, M.; BALLY, F.; BOTTGER, E.C.; BODMER, T.; Rapid identification of mycobacteria to the species leved by polymerase chain reaction and restriction enzyme analysis. Journal of Clinical Microbiology, v.31, n.2, p.175-178,1993.

VAN SOOLINGEN, D.; QUIAN,L. DE HAAS.; P.E.W.; DOUGLAS, J.T.; TRAORE, H.; PORTAELS, F.; QUINA, H.Z.I.; ENKHSAIKAN, D.; NYMADAWA, P.; VAN EMBDEN, J.D.A. Predominante of a single genotype of Mycobacterium tuberculosis in countries of East Ásia. Journal of Clinical Microbiology, v.33, n.3234-3238,1995.

ZANINI, M.S.; MOREIRA, E.C.; LOPES, M.T.P.; OLIVEIRA, R.S.; LEÃO, S.C. FIORAVANTI, R.I.; ROXO, E.; ZUMÁRRAGA, M.; ROMANO, M.I. CATALDI, A.; SALAS, C.E. Mycobacterium bovis : Polymerase Chain Reaction identification in bovine lymphonode biopsies and genotyping in isolates from southeast Brazil by Spolygotyping and Restriction Fragment Length Polymorphism. Memórias do Instituto Osvaldo Cruz, v.96, p.809-813, 2001.

ZINK, A.R.; NERLICH, A.G. Molecular strain identification of the Mycobacterium tuberculosis complex in archival tissue samples. Journal of Clinic Pathology, v.57, p.1185-1192, 2004.

Recebido em $1 / 10 / 08$

Aceito em 11/4/10 